\title{
Une politique qui manque singulièrement de cohérence
}

\author{
Irene Abderhalden, Monique Portner-Helfer, Frank Zobel
}

Addiction Suisse

La politique des addictions est traversée par de multiples incohérences et contradictions. La consommation d'alcool dans l'espace public est perçue comme un problème mais on libéralise davantage le marché de l'alcool. D’un côté, on souhaite élargir l'offre des jeux d'argent sur internet et de l'autre se profile l'abandon des ressources pour la prévention du jeu excessif. Le soutien à la production du tabac reste égal aux moyens attribués à la prévention du tabagisme, et des restrictions plus conséquentes de la publicité, telles qu'elles existent ailleurs en Europe, se heurtent encore à une opposition vigoureuse dans notre pays.

Ces incohérences ne favorisent certainement pas la crédibilité de la politique des addictions en Suisse. Celle-ci semble plutôt être conduite sur la base de positions idéologiques figées et non pas sur la base des connaissances que l'on a de son impact sur les personnes concernées, leur entourage et la société. Il importe donc de revenir à une analyse de la situation en Suisse et sur les enjeux qui lui sont liés.

Les chambres fédérales examinent pour la première fois en parallèle des législations sur l'alcool, sur les produits du tabac et sur les jeux d'argent. C'est une opportunité pour développer une approche crédible et efficace dans le domaine des addictions. D'ailleurs, elles sont aussi interpellées par des villes et cantons sur la question du cannabis. Mais que se passe-t-il vraiment? En réalité, les trois lois sont abordées

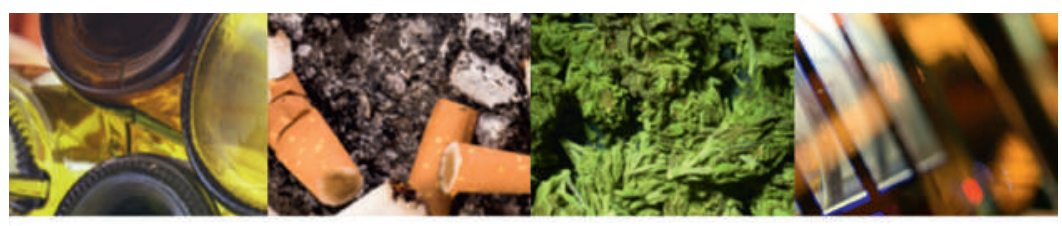

PANORAMA SUISSE DES ADDICTIONS EN 2015

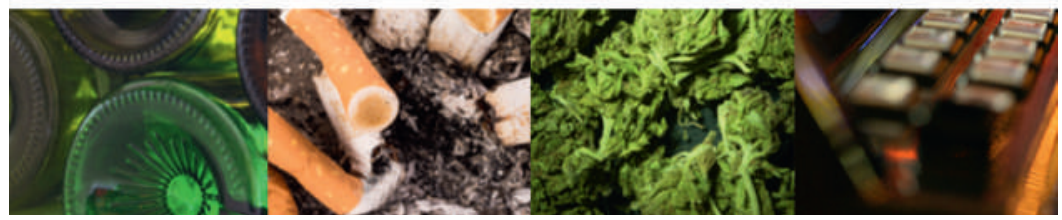

Le logo «Panorama suisse des addictions» illustre la diversité des addictions. comme si elles n'avaient aucun lien et le contenu de chacune des législations fait l'objet de marchandages entre groupes d'intérêts. Il faut alors encore une fois se battre pied à pied pour chaque franc pour la prévention et pour chaque mesure structurelle touchant le prix ou l'accessibilité, dont l'efficacité a pourtant été prouvée.

Pourtant, une autre politique est possible. Elle requiert une acceptation que les problèmes d'addiction ne sont pas ceux d'individus isolés mais aussi ceux de notre société toute entière et de l'environnement que nous créons. Or, les débats publics actuels ignorent jusqu'ici à quel point le marché peut influencer les comportements des individus, notamment ceux qui sont les plus vulnérables. Introduire des limites à la disponibilité et à la promotion de l'alcool, du tabac ou des jeux d'argent ne constitue en fait rien de plus que de réguler un marché qui est de plus en plus laissé à lui-même sans garde-fous. Pour réduire les problèmes d'addiction, on ne peut en effet que développer une régulation du marché efficace et, en parallèle, octroyer des moyens à la prévention, à la réduction des risques et aux soins.

\section{Les addictions au tournant du $\mathrm{XXI}^{\mathrm{e}}$ siècle: un univers en transformation}

Les consommations de substances psychoactives et les autres comportements pouvant engendrer une dépendance ne connaissent pas tous la même évolution. On observe notamment des changements dans les normes sociales pour l'alcool et le tabac, de nouvelles offres pour les jeux d'argent et les drogues illégales, et plus généralement des signes d'une extension des marchés.

\section{Moins d'alcool mais toujours beaucoup d'ivresses}

La dernière décennie a vu une baisse de la consommation d'alcool dans notre pays mais aussi ailleurs. Certains modes de consommation quotidienne se sont ainsi progressivement dissipés et se retrouvent désormais surtout chez les personnes âgées. La conséquence a été une réduction de la consommation 
totale d'alcool, et particulièrement du vin, en Suisse. La baisse de la consommation quotidienne d'alcool des hommes a aussi contribué à un rapprochement entre hommes et femmes. Ces dernières, particulièrement celles âgées entre 65 et 74 ans, boivent aussi plus souvent régulièrement de l'alcool qu'il y a vingt ans.

La consommation totale d'alcool chez les jeunes entre 15 et 24 ans et la consommation à risque a aussi augmenté durant les dernières décennies. Les ivresses ponctuelles stagnent désormais à un haut niveau. L'extension de la durée et de l'offre des loisirs nocturnes, ainsi que la baisse des prix de l'alcool, ont sans doute contribué à cela. La consommation excessive d'alcool n'est toutefois pas l'apanage des jeunes et on la retrouve dans toutes les catégories d'âge. Elle est aussi plus répandue chez les hommes qui ont un emploi et ceux qui disposent d'un revenu élevé.

\section{Le tabac: la baisse est-elle finie?}

L'usage du tabac s'est réduit sous l'effet de mesures structurelles et de transformation des normes sociales. Il n'y a pas si longtemps, on pouvait fumer partout: travail, restaurant ou transports publics. Aujourd'hui, le quart de la population qui fume encore ne le fait souvent que dans la rue et sur les balcons. Les données suggèrent toutefois que la baisse du tabagisme et de l'exposition à la fumée passive pourrait désormais être interrompue.

Drogues illicites: moins d'héroïne, plus d'ecstasy La troisième baisse importante concerne un phénomène qui a causé longtemps d'importants problèmes de santé publique: l'usage, souvent par injection, d'héroïne. Une meilleure prise en charge des usagers a elle aussi contribué à réduire les problèmes liés aux drogues illicites en Suisse. Il reste qu'au moins 40000 jeunes consomment du cannabis chaque jour dont certain du matin au soir. Le marché noir est lui aussi en constante mutation, comme le montre par exemple le retour de l'ecstasy. La vente sur internet, notamment des nouvelles substances psychoactives, se développe également.

\section{Beaucoup de jeux, beaucoup de perdants}

L'offre de jeux d'argent en Suisse a massivement augmenté au début de ce siècle avec l'introduction des maisons de jeu et la diversification des jeux de loterie. Aujourd'hui, la Suisse dispose de l'une des densités de casinos les plus élevées au monde. Elle souhaite aussi élargir l'offre des jeux d'argent sur internet.
Le nombre de joueurs excessifs et les coûts sociaux associés aux jeux d'argent sont eux aussi conséquents et la recherche a déjà montré que la pratique des jeux en ligne est plus dangereuse qu'ailleurs.

\section{Certains comportements changent} mais la plupart des dommages restent

Malgré des évolutions positives dans plusieurs domaines, les dommages restent élevés. Le tabac reste la première cause de mortalité précoce en Suisse et l'alcool figure en troisième position. Au total, les substances psychoactives et le jeu excessif sont associés chaque année à plus de 10000 décès et à des coûts sociaux supérieurs à 10 milliards de francs. Il faut aussi mentionner les souffrances de plusieurs centaines de milliers d'individus dépendants et de leur entourage ou encore les nuisances, surtout liées à l'alcool, qui touchent plus de la moitié de la population.

\section{Des mesures structurelles sont nécessaires}

La révision actuelle de la Loi sur l'alcool offre une chance réelle de réduire la consommation problématique. Des mesures parfaitement réalisables et dont l'efficacité a été prouvée, comme une hausse des prix et des limitations d'horaires de vente, sont des mesures nécessaires pour réguler le marché. Pour la nouvelle Loi sur les produits du tabac, Addiction Suisse se prononce pour une interdiction nationale de la vente aux mineurs strictement contrôlée, ainsi que pour une interdiction totale de la publicité et du parrainage. Cela doit être assorti d'une interdiction des automates à cigarettes ainsi que des incitations à la vente sous forme de rabais. En ce qui concerne le débat sur les jeux d'argent, nous demandons une meilleure protection des joueurs, une interdiction des jeux gratuits et une régulation durable de la publicité. Les cantons doivent aussi avoir la garantie qu'ils recevront l'argent nécessaire pour la prévention et le traitement du jeu excessif, afin qu'ils puissent accomplir la mission que leur confère le projet de loi. Addiction Suisse salue par ailleurs les discussions actuelles dans les villes au sujet de modèles de régulation du marché du cannabis, en particulier le plus abouti à Genève. L'évaluation scientifique d'un accès légal au cannabis pour les adultes, dans le cadre de coopératives de consommation, permettra de mieux connaître les coûts et bénéfices sanitaires de telles approches. 